\title{
Ventricular Tachycardia in a Patient Undergoing Proximal Right Coronary Artery Stenting-Importance of Lesion Pre Dilatation.
}

\author{
Dr Venkatesh Krishnamurthy Tekur, \\ Consultant Interventional Cardiologist, Apollo Hospitals, Opp. IIM, Bannerghatta Road, \\ Bangalore Karnataka State, India.
}

\begin{abstract}
Ventricular tachycardia due to occlusive engagement of right coronary artery by different types of coronary catheters have been known. The authors describe here a case of ventricular tachycardia occurring when a stent was being positioned in the proximal right coronary artery after uneventful engagement of a right coronary ostium by a Judkins $6 F$ Guide. It is presumed that direct stent placement across the lesion without predilating the lesion could have caused occlusion of the right coronary artery lumen with consequent myocardial hypoxia and ventricular arrhythmia.

Keywords: Ventricular Tachycardia, Right Coronary Artery, Direct Stenting

Key-Messages : Direct stent placement across the lesion without predilating the lesion in proximal right coronary artery can cause occlusion of the right coronary artery lumen with consequent hypoxia and ventricular arrhythmia.
\end{abstract}

\section{Case History}

A 52 -year lady was admitted with symptoms of retrosternal chest pain suggestive of unstable angina with ECG showing ST-Segment depressions in inferior leads. She underwent a coronary angiogram which showed significant and discreet $80 \%$ stenosis of proximal right coronary artery without calcification. A $5 \mathrm{~F}$ right Judkins catheter was used to engage the right coronary ostium during diagnostic angiogram via right femoral approach. The diagnostic coronary angiogram was carried out uneventfully without any evidence of pressure damping on engagement of the right coronary artery.[FIG 1]. A direct stenting of the proximal right coronary artery lesion was planned. A right $6 \mathrm{~F}$ Judkins Guide catheter was used to engage the right coronary artery .There was no catheter induced pressure damping after engagaement of the right coronary ostia by the right Judkins guide. A BMW PCI wire was used to cross the lesion in the right coronary artery. A $3.5 \mathrm{X} 14 \mathrm{~mm}$ Xience Xpedition stent was advanced across the lesion and contrast was injected prior to final deployment of the stent.Even before the stent could be deployed, patient developed sudden ventricular tachycardia.[FIG 2]. The right Judkins guide was immediately disengaged from the coronary ostia and the arrhythmia was electrically cardioverted with 200J DC shock. Subsequently, the lesion was stented uneventfully after pre- dilataion of the lesion with $2.5 \times 12 \mathrm{~mm}$ semi-compliant balloon taking care to keep the guide disengaged during balloon dilatation.[FIG 3].TIMI Grade 3 flow was established with good results.[FIG 4].

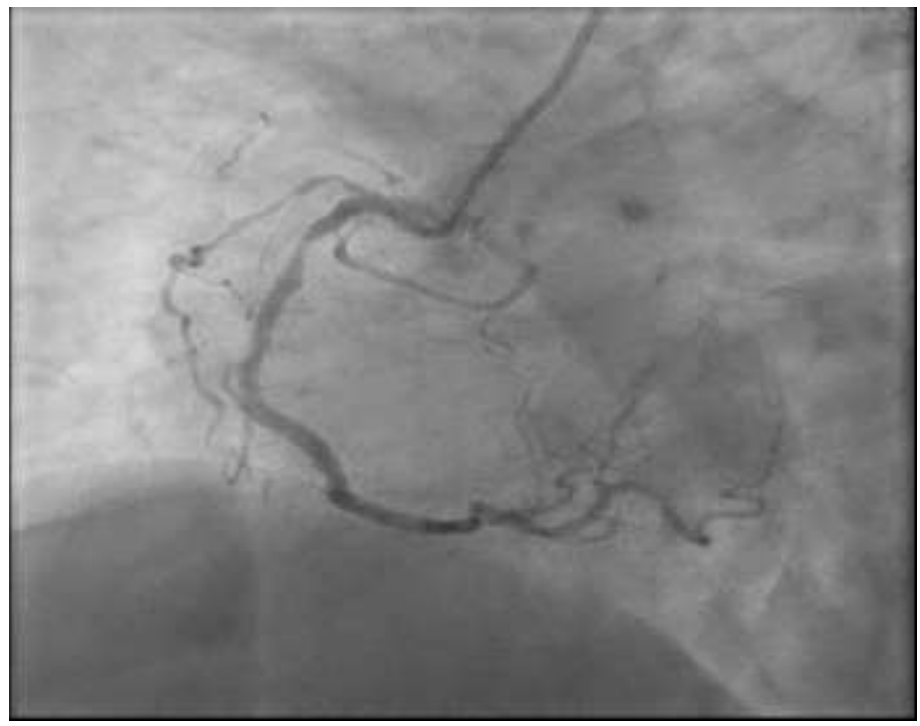

Fig 1. Diagnostic right coronary angiogram showing $80 \%$ stenosis of proximal RCA. 


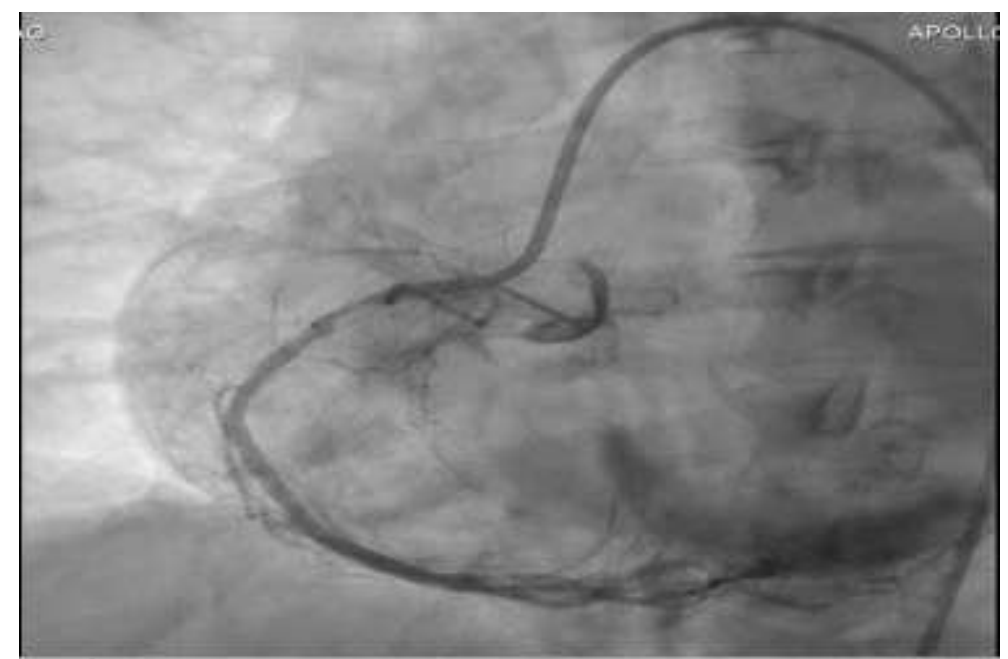

Fig 2. Patient developed ventricular tachycardia with contrast hold up in right coronary artery during injection for positioning of stent

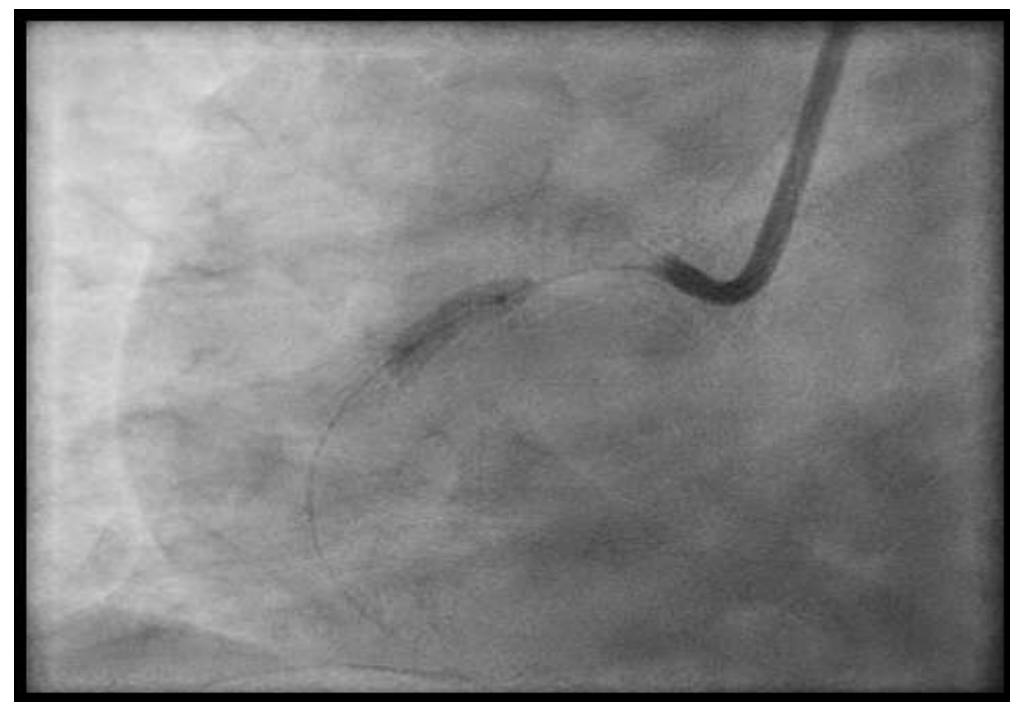

Fig 3. Lesion Predilated with $2.5 \times 12 \mathrm{~mm}$ semi compliant balloon after disengaging right Judkins Guide.

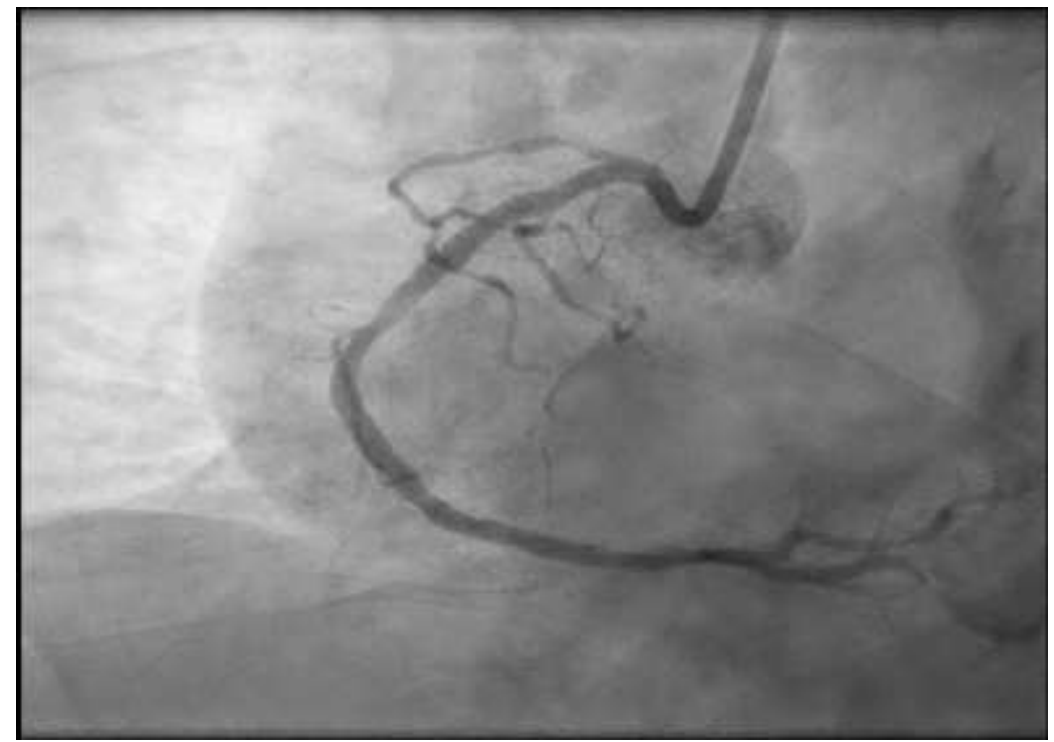

Fig 4. TIMI Grade 3 Flow established with good result. 


\section{Discussion}

Engaged coronary artery catheter pressures should always be checked before injecting contrast medium. Injection of contrast with damped or ventricularized waveforms can cause dissections of the artery or arrhythmias. In one study, of the 24 episodes of ventricular arrhythmia, 14 were related to catheter manipulation, 8 to ischemia, and 2 to the contrast medium, while the cause could not be clearly established in $4{ }^{1}$ .The episodes of ventricular arrhythmias are more frequent during right coronary artery procedures than left coronary artery procedures ${ }^{2}$. Early reports suggested that, ventricular arrhythmias could be caused by ischemia or mechanical complications or contrast medium toxicity ${ }^{3,4}$. Ischemia and contrast medium could make myocardium more susceptible to ventricular arrhythmias and certain transient events such as improper manipulation and acute ischemia could play an important role in triggering ventricular tachycardia attack. Some of the episodes of ventricular arrhythmias may involve multiple mechanisms. In this patient, placement of the coronary stent across the lesion prior to its deployment could have seriously compromised the lumen causing myocardial hypoxia and subsequent injection of contrast media to ascertain the stent's position pre deployment could have triggered the serious ventricular tachycardia. The same stent was successfully deployed uneventfully after pre dilating the coronary lesion with a semi compliant balloon taking care to keep the right coronary artery guide disengaged.

\section{References}

[1]. Ventricular Arrhythmia Onset During Diagnostic Coronary Angiography With a 5F or 4F Universal Catheter.Jun Chen, LiJian Gao, Min Yao, Jilin Chen. Rev Esp Cardiol. 2008;61:1092-5 - Vol. 61 Num.10 DOI: 10.1016/S1885-5857(09)60013-X.

[2]. Nishimura RA, Holmes DR, McFarland TM, Smith HC, Bove AA. Ventricular arrhythmias during coronary angiography in patients with angina pectoris or chest pain syndromes. Am J Cardiol. 1984;53:1496-9.

[3]. Murdock DK, Euler DE, Becker DM, Murdock JD, Scanlon PJ, Gunnar RM. Ventricular fibrillation during coronary angiography: an analysis of mechanisms. Am Heart J. 1985;109:265-73.

[4]. Morris TW. A review of coronary arteriography and contrast media induced ventricular fibrillation. Acta Radiol Suppl. 1995;399: $100-4$. 